\title{
Design of a stable state feedback controller based on the multirate sampling of the plant output
}

\author{
$\operatorname{AUTHOR}(\mathrm{S})$ :
}

Hagiwara, T; Araki, M

\section{CITATION:}

Hagiwara, T ... [et al]. Design of a stable state feedback controller based on the multirate sampling of the plant output. IEEE Transactions on Automatic Control 1988, 33(9): 812819

\section{ISSUE DATE:}

1988-09

URL:

http://hdl.handle.net/2433/39963

\section{RIGHT:}

(c) 1988 IEEE. Personal use of this material is permitted. However, permission to reprint/republish this material for advertising or promotional purposes or for creating new collective works for resale or redistribution to servers or lists, or to reuse any copyrighted component of this work in other works must be obtained from the IEEE. 


\title{
Design of a Stable State Feedback Controller Based on the Multirate Sampling of the Plant Output
}

\author{
TOMOMICHI HAGIWARA AND MITUHIKO ARAKI, MEMBER, IEEE
}

\begin{abstract}
This paper proposes a new type of controller which detects the $i$ th plant output $N_{i}$ times during a period $T_{o}$ and changes the plant inputs once during $T_{o}$. It will be shown that an arbitrary state feedback can be realized by such controllers if the plant is observable. This implies, for instance, that arbitrary symmetric pole assignment is possible if the plant is controllable. It is also shown that, if the plant has no zeros at the origin, the state transition matrix of the controller itself can be set arbitrarily without changing the state feedback to be realized. That is to say, inversely expressed, any state feedback can be equivalently realized by a controller with any prescribed degree of stability.
\end{abstract}

\section{INTRODUCTION}

TN the design of controllers based on the state-space method, Lobservers are often used to estimate inaccessible elements of the state vector. The advantage of using an observer exists in the fact that the observer design can be, at least superficially, separated from the regulator design and, therefore, the whole designing procedure is simplified. Nonetheless, we can point out two clear disadvantages which accompany the introduction of an observer; namely, increase of the order of the system and possibility of producing an unstable controller [1], [2]. Especially, an unstable controller is evidently unwelcome from the viewpoint of stability margins and integrity, and is said to deteriorate the tracking ability [3]. Therefore, it is desired to find new controllers which are stable, and, at the same time, are capable of assigning arbitrary dynamical characteristics to the closed-loop system.

Recently, much research was reported which intended to solve the above problem by using digital controllers. Namely, Chammas and Leondes proposed to use a certain type of periodically time-varying gain controller [4]-[7], the present authors proposed the multirate-input controllers [8], [9], Greschak and Verghese, and Khargonekar et al. proposed another type of periodically time-varying controller [10], [11], and Mita et al. proposed the intersample data controllers [12]. All these controllers have the common feature that they include periodically time-varying elements. For the convenience of explanation, we introduce the term " frame period $T_{o}$ " to refer to the "cycle" of the controllers, and use the term "sampling period" to indicate the interval with which the plant inputs are changed or the plant outputs are detected. The contributions and limitations of the above-mentioned research are as follows, where controllability and observability of the plant are accepted as the basic assumption.

Chammas and Leondes made studies about the ability of the controllers which use periodically time-varying gains [4]-[7]. Especially, in their third report [6], they considered the class of controllers which detect all the plant outputs once in a frame period $T_{o}$ and change all the feedback gains (as a result, all the plant inputs) $N$ times in $T_{o}$ with uniform sampling periods, and showed that arbitrary symmetric pole assignment can be realized

Manuscript received May 28, 1987; revised February 19, 1988. Paper recommended by Associate Editor, B. H. Krogh. This work was supported in part by a Grant-in-Aid for Scientific Research C60550294.

The authors are with the Department of Electrical Engineering, Kyoto

The authors are with
University, Kyoto, Japan.

IEEE Log Number 8822361. by such controllers if $N$ is chosen equal to or greater than the controllability index of the plant. The present authors tried to clarify the ability of the multirate sampled-data controllers [13][21], especially those controllers which had been treated in [20], [21]. As one of the simplest cases, they considered the multirateinput controllers which detect all the plant outputs once in a frame period $T_{o}$ and change the $i$ th plant input $N_{i}$ times in $T_{o}$ with uniform sampling periods, and showed that arbitrary symmetric pole assignment can be realized by such controllers if $\left(N_{1}, \cdots\right.$, $N_{m}$ ) is chosen equal to or greater, in the elementwise sense, than the controllability Kronecker invariants [8], [9]. Chammas and Leondes' controllers given in [6] are the special case of the controllers of [8] and [9] in which $N_{1}=\cdots=N_{m}=N$. The controllers of [6], [8], and [9] use only gain feedback and, therefore, are always stable. So, the above results imply that arbitrary symmetric pole assignment can be realized by a certain class of "stable" digital controllers, and thus, give a solution to the problem presented in the first paragraph. When the closedloop poles are chosen, the feedback gains can be computed by using an ordinary pole assignment program for the time-invariant state feedback controllers together with a matrix inversion program; namely, computational efforts required in the design of the above class of controllers are almost the same as those required for ordinary time-invariant controllers. Thus, the controllers of [6], [8], and [9] have advantages for applications in three aspects: they are always stable, can assign arbitrary symmetric poles, and do not require much computational effort for design. But, unfortunately, they have a serious drawback in the waveforms. Namely, in the simulation study, it is observed that the plant inputs tend to take large positive values and large negative values alternatively for several times at the early stage of the transient response, especially when the order of the plant is large as compared to the number of the inputs. This tendency can be interpreted as the natural consequence of the basic control strategy of this class of controllers that make use of frequent change of inputs to regulate the state. Because of this drawback, the controllers of [6], [8], and [9] cannot be strongly recommended for industrial application at the present stage.

Khargonekar et al. [11] treated periodically time-varying controllers, which are different from those of [6], [8], and [9] in the way of detecting the plant outputs, i.e., the former detect the plant outputs $N$ times in a frame period $T_{o}$ whereas the latter detect only once in $T_{o}$. A special class of such time-varying controllers had been already studied by Greschak and Verghese [10]. Khargonekar et al, established one-to-one correspondence between their time-varying controllers and time-invariant controllers defined on extended signal spaces, and studied the robust stabilizability, the strong stabilizability, the simultaneous stabilizability, and the sensitivity minimization problems. Their research includes several enlightening ideas, but their results are not enough to be applied to industrial problems directly at the present stage. There are three reasons for that. First, Khargonekar et al.'s design procedure is much more complicated than those of [6], [8], and [9]. Secondly, it is not known whether their controllers can always assign arbitrary poles to the closed-loop system and still remain stable. Khargonekar et al. did not make any comments about these two points, but Greschak and Verghese's result [10] 
illustrates the situation very well. Greschak and Verghese treated the special class of the Khargonekar et al. type controllers which consist solely of $\mathrm{N}$-periodic gains and studied the pole assignment problem for scalar plants. They showed that arbitrary symmetric pole assignment is possible by their controller if the plant is of second order, if its pulse transfer function satisfies a certain condition, ${ }^{1}$ and if $N=3$. But, they did not reach any concrete results in the cases of third and higher order plants. That was mainly because the coefficients of the closed-loop characteristic polynomials become too complicated for the analysis of pole assignability when the order of the plant and the frequency of change of the gains become large. This implies that computational efforts required for precise design are enormous. In addition, the fact that " $N=3$ " is required for the second-order scalar plants suggests that the Greschak and Verghese's controllers possess weaker pole-assigning ability than those of [6], [8], and [9]. (Note that the controllers of [6], [8], and [9] can realize arbitrary pole assignment with " $N=2$ " for second-order scalar plants.) Khargonekar et al. raised the ability of the controllers by introducing dynamical structures, but the problem of the computational difficulties seems to remain unchanged, and it is not still clear whether arbitrary symmetric pole assignment is always possible by stable controllers. The third reason why Khargonekar et al.'s results are not enough for applications is the problem of the input waveforms. Namely, their controllers also use the control principle of regulating the state by frequent change of controller dynamics. Therefore, there is a possibility that their controllers also exhibit the phenomena that the plant inputs change very rapidly at the early stage of the transient response. Unfortunately, this point has not been studied because of the computational difficulties mentioned above. To summarize Khargonekar et al.'s controllers cannot be strongly recommended for applications at the present stage, either, because computational efforts for design are very large, arbitrary symmetric pole assignment cannot be achieved with the present knowledge, and there is a possibility that rapid change of inputs may occur.

Mita et al.'s research has a different feature; namely, they considered using a specific set of intersample output data for control instead of changing inputs frequently, and showed that controllable and observable plants with no zeros at the origin are always stabilizable by stable controllers using the intersample output data [12]. The result can be extended up to symmetric pole assignability although it was not mentioned explicitly. However, their result suffers from two strong limitations. First, it can be applied only to scalar plants and to a limited class of multivariable plants (which they call "mechanical systems") but not to general multivariable systems. Secondly, their controller becomes an $n$ dimensional dynamical system, where $n$ is the dimension of the state of the plant, and this dimension cannot be decreased as long as their design method is used.

From the previous research, it is clear that introduction of periodically time-varying elements brings about qualitative improvement of the controllers' ability. However, the available results are not enough for applications as discussed above. So, in this paper, we propose a new class of periodically time-varying controllers, i.e., we propose "multirate-output controllers" (abbreviated as MROC's), which are the dual of the multirateinput controllers proposed in [8] and [9]. The MROC's change the plant inputs once in a frame period $T_{o}$ and detect the $i$ th plant ou:put $N_{i}$ times in $T_{o}$ with uniform sampling periods. It will be shown in the following that MROC's with satisfactorily large $N_{i}$ 's can equivalently realize arbitrary state feedback if:

Al) The plant is observable.

The fact that the arbitrary state feedback can be equivalently

' Let the pulse transfer function of the plant be $\left(\beta_{1} z+\beta_{2}\right) /\left(z^{2}+\alpha_{1} z+\right.$ $\alpha_{2}$ ). They required that i) $\beta_{2} \neq 0$ and ii) $\alpha_{1}$ and $\beta_{1}$ are not simultaneously zero. They also derived the necessary and sufficient condition so that the second order plants become stable-pole assignable by controllers which change gains twice (i.e., $N=2$ ) during a frame period. realized implies that arbitrary symmetric pole assignment and LQ optimal feedback can be achieved if:

A2) The plant is controllable.

In addition, it will be shown that the state transition matrix of the controller itself can be chosen freely (independently of the choice of the state feedback) if:

A3) The plant has at least as many outputs as inputs and does not have invariant zeros at the origin.

Here the state transition matrix of the controller is defined for the frame period $T_{o}$. The design of the MROC's can be carried out following the next procedure.

DI) Choose the closed-loop poles to be assigned (or alternatively, quadratic performance index), and calculate the state feedback matrix $F$ which realizes those poles (or minimizes the given performance index).

D2) Choose the state transition matrix $M$ of the controller.

D3) Solve a linear simultaneous equation. Then, we obtain the actual parameters of the MROC.

In step D1), we can use the standard routine of pole assignment by state feedback (or the LQ optimization routine). So, necessary computational efforts for design are almost the same as those required for the design of ordinary time-invariant controllers. Concerning the waveforms, the results of simulation indicate that rapid change of the inputs does not occur even if the order of the plant is large compared to the number of inputs and outputs. This can be interpreted as the consequence of the new control principle employed by MROC's. Namely, MROC's make use of frequent detection of plant outputs for equivalently realizing the specified state feedback, and, therefore, do not need to change the plant inputs rapidly.

To summarize, MROC's have the following advantages: first, they have the same ability as the state feedback in adjusting the closed-loop characteristics of the control systems; second, they have the ability of choosing arbitrary state transition matrices of the controllers themselves; third, the above two results apply to a wide class of plants (i.e., observable and controllable plants which have at least as many outputs as inputs and which do not have invariant zeros at the origin); fourth, computational efforts required in the design procedure are almost the same as those required for the ordinary time-invariant controllers; and fifth, they do not change the plant inputs rapidly as the multirate-input controllers and other types of controllers which use frequent changes of gains for regulation. Thus, MROC's are much more suited to industrial applications than the periodically time-varying controllers proposed before [4]-[12]. The remaining important item, which should be clarified before MROC's are applied, is the robustness problem. In an example studied in Section V, it will be shown that the stability margins seen at the input terminal change according to the state transition matrix of the controller and can be set at satisfactory values by adjusting the controller dynamics. It can be generally shown that the same stability margins as the direct state feedback are realized when the state transition matrix of the controller is set to zero. (This result will be reported as a separate paper [30].) These results suggest that MROC's have the ability of assuring satisfactory robustness, too.

\section{Multirate-Output Sampling Mechanism and Preliminary} RESULTS

Consider the linear time-invariant continuous-time plant described by

$$
\begin{gathered}
\frac{d}{d t} x(t)=A x(t)+B u(t) \\
y(t)=C x(t)
\end{gathered}
$$

where the state $x \in R^{n}$, the plant input $u \in R^{m}$, and the plant output $y \in R^{p}$. Connecting a sampler and a zero-order hold with 


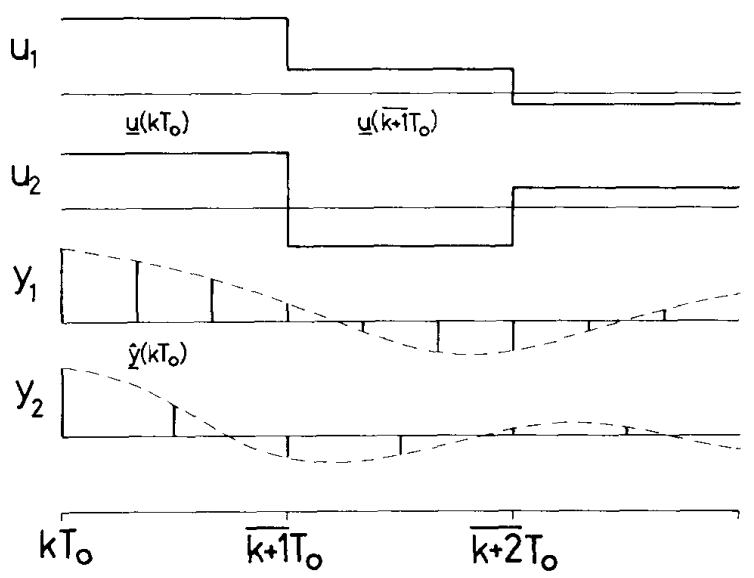

Fig. 1. Multirate-output sampling mechanism. $\left(m=p=2, N_{1}=3\right.$,

$$
N_{2}=2 \text { ). }
$$

the sampling period $T_{o}$ to each plant input, $u(t)$ is given by

$$
\boldsymbol{u}(t)=u\left(k T_{o}\right) \quad\left(k T_{o} \leqq t<\overline{k+1} T_{o}\right)
$$

Substituting (3) into (1) and solving (1), we obtain

$$
x\left(k T_{o}+s\right)=\exp (A s) x\left(k T_{o}\right)+\int_{0}^{s} \exp (A t) B d t \cdot u\left(k T_{o}\right)
$$

for $0 \leqq s \leqq T_{o}$. Detecting the $i$ th plant output $y_{i}$ at every $T_{i}$, the sampled value is given by

$$
y_{i}\left(k T_{o}+\mu T_{i}\right)=c_{i} x\left(k T_{o}+\mu T_{i}\right) \quad\left(\mu=0, \cdots, N_{i}-1\right) .
$$

Here, $c_{i}$ is the $i$ th row of the output matrix $C$, and $T_{i}$ and $T_{o}$ are assumed to be related by

$$
T_{i}=T_{o} / N_{i} \quad(i=1, \cdots, p)
$$

where $N_{i}$ is a positive integer. Equation (6) implies that the $i$ th plant output is detected $N_{i}$ times during $T_{o}$ (Fig. 1). We refer to $T_{o}$ as the input sampling period or the frame period (in the following, we mainly use the latter terminology; the reason why $T_{o}$ has two names will become clear in the next section), $T_{i}$ as the $i$ th output sampling period, $\left(N_{1}, \cdots, N_{p}\right)$ as the output multiplicities, and the above sampling mechanism as the multirate-output sampling mechanism. Next, several properties of the multirate-output sampling mechanism will be derived.

Put $s=T_{o}$ in (4) to obtain

$$
\boldsymbol{x}\left(\overline{k+1} T_{o}\right)=\exp \left(A T_{o}\right) \boldsymbol{x}\left(k T_{o}\right)+\int_{0}^{T_{o}} \exp (A t) B d t \cdot u\left(k T_{o}\right)
$$

Solving the above equation for $x\left(k T_{o}\right)$ and substituting the solution into (4), we obtain

$$
\begin{aligned}
x\left(k T_{o}+s\right)=\exp \left\{A\left(s-T_{o}\right)\right\} & x\left(\overline{k+1} T_{o}\right) \\
& +\int_{0}^{s-T_{o}} \exp (A t) B d t \cdot u\left(k T_{o}\right)
\end{aligned}
$$

Substituting the above equation into (5), we obtain

$$
\begin{aligned}
& y_{i}\left(k T_{o}+\mu T_{i}\right)=c_{i} \exp \left\{A\left(\mu T_{i}-T_{o}\right)\right\} x\left(\overline{k+1} T_{o}\right) \\
& \quad+c_{i} \int_{0}^{\mu T_{i}-T_{o}} \exp (A t) B d t \cdot u\left(k T_{o}\right) \quad\left(\mu=0, \cdots, N_{i}-1\right)
\end{aligned}
$$

Moving the terms of $x\left(\overline{k+1} T_{o}\right)$ to the left-hand side, moving the terms of $y_{i}\left(k T_{o}+\mu T_{i}\right)$ to the right, and expressing the equations for $i=1, \cdots, p ; \mu=0, \cdots, N_{i}-1$ by a vector-matrix form, we obtain

$$
\hat{C} \boldsymbol{x}\left(\overline{k+1} T_{o}\right)=\hat{y}\left(k T_{o}\right)-\hat{G} u\left(k T_{o}\right) \quad(k \geqq 0) .
$$

Here, $\hat{C} \in R^{\bar{N} \times n}, \hat{G} \in R^{\bar{N} \times m}$, and $\hat{y}\left(k T_{o}\right) \in R^{\hat{N}}$ are, respectively, given by

$$
\hat{C}=\left\{\begin{array}{c}
c_{1} \exp \left(-A N_{1} T_{1}\right) \\
: \\
c_{1} \exp \left(-A T_{1}\right) \\
: \\
c_{p} \exp \left(-A N_{p} T_{p}\right) \\
: \\
c_{p} \exp \left(-A T_{p}\right)
\end{array}\right\},
$$

$$
\hat{G}=\left(\begin{array}{cc}
c_{1} \int_{0}^{-N_{1} T_{1}} & \exp (A t) B d t \\
c_{1} \int_{0}^{-T_{1}} & \exp (A t) B d t \\
c_{p} \int_{0}^{-N_{p} T_{p}} \exp (A t) B d t \\
c_{p} \int_{0}^{-T_{p}} \exp (A t) B d t
\end{array}\right],
$$

and

$$
\hat{y}\left(k T_{o}\right)=\left\{\begin{array}{c}
y_{1}\left(k T_{o}\right) \\
\vdots \\
y_{1}\left(k T_{o}+\overline{N_{1}-1} T_{1}\right) \\
\vdots \\
y_{p}\left(k T_{o}\right) \\
\vdots \\
y_{p}\left(k T_{o}+\overline{N_{p}-1} T_{p}\right)
\end{array}\right\}
$$

where $\bar{N}$ is given by

$$
\bar{N}=\sum_{i=1}^{p} N_{i}
$$

The vector $\hat{y}\left(k T_{o}\right)$ is composed of the multirate-sampled-data of the outputs in the interval of a single frame period, and its relation to the inputs and the final state in that interval is given by (10). We refer to (10) as the basic formula of the multirate-output sampling mechanism. We can show that the coefficient matrix $\hat{C}$ and the pair $[\hat{C} \hat{G}]$ can be made to have full ranks by choosing $N_{i}$ 's sufficiently large. To facilitate the statement of the results, we introduce the term "observability index vector."

Definition (Observability Index Vector): Consider an observable pair $(A, C)$ where $A \in R^{n \times n}$ and $C \in R^{p \times n}$. Express $C$ as $C=\left[c_{1}^{T}, \cdots, c_{p}^{T}\right]^{T}$. A set of $p$ integers $\left(n_{1}, \cdots, n_{p}\right)$ is said to be an observability index vector (abbreviated as OIV) of the pair $(A, C)$ if

$$
\sum_{i=1}^{p} n_{i}=n
$$


and the vectors $c_{1}, \cdots, c_{1} A^{n_{1}-1} ; c_{2}, \cdots, c_{2} A^{n_{2}-1} ; \cdots ; c_{p}, \cdots$, $c_{p} A^{n_{p}-1}$ are linearly independent. Here, $n_{i}=0$ means that the vectors of the form $c_{i} A^{v}$ do not appear in the above series.

Note that an observable pair has at least one OIV [i.e., the set of Kronecker invariants [22] of the pair $\left(A^{T}, C^{T}\right)$ ] and may have more OIV's if $p \geqq 2$. An algorithm to find OIV's was given by Luenberger [23]. Also note that any OIV is preserved under the similarity transformation.

Concerning the matrix $\hat{C}$ of the basic formula (10), we have the next result.

Lemma 1: Let $(A, C)$ be an observable pair. Then, the matrix $\hat{C}$ given by (11) has full column rank $(=n)$ for almost every frame period $T_{o}$ if the output multiplicities $\left(N_{1}, \cdots, N_{p}\right)$ satisfy

$$
N_{i} \geqq n_{i} \quad(i=1, \cdots, p)
$$

where $\left(n_{1}, \cdots, n_{p}\right)$ is an OIV of the pair $(A, C)$.

In the above lemma, and also in the following, the statement "for almost every $T_{o}$ "' is used to shortly express the fact that the assertion fails only at isolated values of $T_{o}$. The proof of Lemma 1 is given in the Appendix. Concerning the pair $[\hat{C} \hat{G}]$, we have the next result.

Lemma 2: Let $(A, C)$ be an observable pair, and suppose that

$$
\operatorname{rank}\left(\begin{array}{cc}
A & B \\
C & 0
\end{array}\right)=n+m \text {. }
$$

Then, the matrix $[\hat{C} \hat{G}]$ given by (11) and (12) has full column rank $(=n+m)$ for almost every frame period $T_{o}$ if the output multiplicities $\left(N_{1}, \cdots, N_{p}\right)$ satisfy

$$
N_{i} \geqq m_{i} \quad(i=1, \cdots, p)
$$

where $\left(m_{1}, \cdots, m_{p}\right)$ is an OIV of the augmented system

$$
\left(\left(\begin{array}{cc}
A & B \\
0 & 0
\end{array}\right),\left[\begin{array}{ll}
C & 0
\end{array}\right)\right. \text {. }
$$

Proof: Observing that

$$
\exp \left(\begin{array}{cc}
A & B \\
0 & 0
\end{array}\right) t=\left(\begin{array}{cc}
\exp (A t) & \int_{0}^{t} \exp (A \tau) B d \tau \\
0 & I
\end{array}\right)
$$

it is easy to see that the matrix $[\hat{C} \quad \hat{G}]$ given by $(11)$ and $(12)$ has the same structure as the matrix $\hat{C}$ if $(A, C)$ is replaced by the pair (19) of the coefficient matrices of the augmented system. Since the augmented system is observable if and only if two assumptions of the lemma are satisfied [24], the result immediately follows from Lemma 1.

Q.E.D.

\section{Multirate-Output Controller and Equivalent State FEEDBACK}

Consider controllers which include the multirate-output sampling mechanism given in the last section, and which determine the control input by

$$
\boldsymbol{u}\left(\overline{k+1} T_{o}\right)=\boldsymbol{M u}\left(k T_{o}\right)-H \hat{y}\left(k T_{o}\right)
$$

where $M \in R^{m \times m}$ and $H \in R^{m \times \bar{N}}$. In view of (13), the above equation means that the control inputs for the $(k+1)$ th frame period are determined based on the values of the control inputs for the $k$ th frame period and the sampled-data of the outputs obtained during the $k$ th frame period. Note that the above controller is an $m$ th-order discrete-time dynamical system whose state is $u\left(k T_{o}\right)$. We refer to the above type of controllers as the multirate-output controllers (abbreviated as MROC's). MROC's can be regarded as a special class of multirate sampled-data controllers in which the input sampling periods are uniform and the same as the frame period.

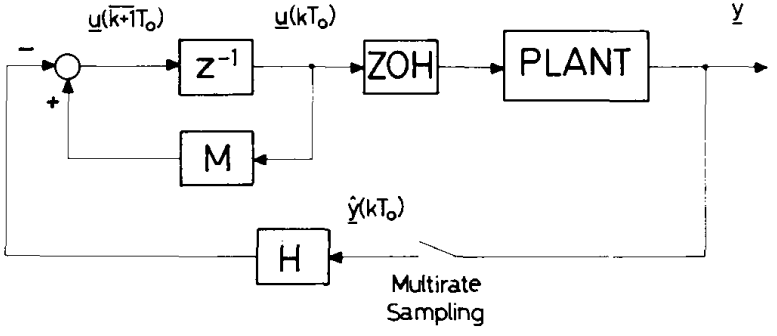

Fig. 2. Closed-loop configuration with a multirate-output controller.

We have the following theorem which tells that an arbitrary state feedback can be equivalently realized by MROC's.

Theorem 1: Suppose that $(A, C)$ is an observable pair and that the output multiplicities $\left(N_{1}, \cdots, N_{p}\right)$ satisfy

$$
N_{i} \geqq n_{i} \quad(i=1, \cdots, p)
$$

where $\left(n_{1}, \cdots, n_{p}\right)$ is an OIV of the pair $(A, C)$. Then, for almost every frame period $T_{o}$, we can make the control law (20) equivalent to any state feedback control law

$$
\boldsymbol{u}\left(k T_{o}\right)=-\boldsymbol{F x}\left(k T_{o}\right) \quad(k \geqq 1)
$$

by suitable choice of the matrices $H \in R^{m \times \bar{N}}$ and $M \in R^{m \times m}$.

Proof: Multiplying the basic formula (10) of the multirateoutput sampling mechanism by a matrix $H$ from the left, we obtain

$$
H \hat{C} x\left(\overline{k+1} T_{o}\right)=H \hat{y}\left(k T_{o}\right)-H \hat{G} u\left(k T_{o}\right) .
$$

Therefore, the control law (20) becomes equivalent to the state feedback (22) if the matrix $H$ satisfies

$$
H \hat{C}=F
$$

and if we set

$$
M=H \hat{G} .
$$

By Lemma 1, there exists a matrix $H$ which satisfies (24) for any $F \in R^{m \times n}$. This completes the proof.

If we determine the matrices $H$ and $M$ by (24) and (25), the resulting closed-loop system becomes as shown in Fig. 2, and the stability of the controller itself is determined by the matrix $H \hat{G}$. Since an unstable controller always provides the closed-loop system with some additional unstable zeros beyond those of the plant, it is desirable to use a stable controller from the viewpoint of sensitivity to disturbances and ability to track reference inputs [3]. So, let us study whether $H \hat{G}$ can be made stable (in the discrete-time sense) or not.

First, suppose that the output multiplicities $\left(N_{1}, \cdots, N_{p}\right)$ are set to the minimum values satisfying (21), i.e.,

$$
N_{i}=n_{i} \quad(i=1, \cdots, p) .
$$

Then, the matrix $\hat{C}$ becomes a nonsingular square matrix and the $H$ satisfying (24) is uniquely determined by

$$
H=F \hat{C}^{-1} .
$$

This means that the stability of the matrix $H \hat{G}$ depends solely on the choice of the state feedback matrix $F$ in this case.

Next consider the case where the output multiplicities $\left(N_{1}, \cdots\right.$, $N_{p}$ ) are set larger than the minimum values as

$$
N_{i}>n_{i} \text {. }
$$

Then, we can find, in general, infinitely many matrices $H$ which satisfy (24), and it becomes plausible that we can select $H$ so that 
the matrix $H \hat{G}$ becomes stable. Concerning this point, we have the following theorem.

Theorem 2: Suppose that $(A, C)$ is an observable pair and that

$$
\operatorname{rank}\left(\begin{array}{cc}
A & B \\
C & 0
\end{array}\right)=n+m
$$

Further suppose that the output multiplicities $\left(N_{1}, \cdots, N_{p}\right)$ satisfy

$$
N_{i} \geqq m_{i} \quad(i=1, \cdots, p)
$$

where $\left(m_{1}, \cdots, m_{p}\right)$ is an OIV of the augmented system (19). Then, for almost every frame period $T_{o}$, there exists a matrix $H$ $\in R^{m \times N}$ such that

$$
H \hat{C}=F
$$

and

$$
H \hat{G}=M
$$

where $F \in R^{m \times n}$ is an arbitrarily specified matrix corresponding to the desired state feedback and $M \in R^{m \times m}$ is an arbitrarily specified matrix corresponding to the desired state transition matrix of the controller itself.

Proof: Note that (31) and (32) are equivalent to

$$
H\left[\begin{array}{ll}
\hat{C} & \hat{G}
\end{array}\right]=\left[\begin{array}{ll}
F & M
\end{array}\right] .
$$

By Lemma 2, (33) has a matrix solution $H$ if the assumptions of the theorem are satisfied.

Q.E.D.

The above theorem implies that we can equivalently realize any state feedback $F$ by a multirate-output controller possessing any prescribed degree of stability since we can choose the matrix $M$ arbitrarily. In particular, if $M=0$, the control law (20) reduces to the multirate sampled-data nondynamic output feedback

$$
u\left(\overline{k+1} T_{o}\right)=-H \hat{y}\left(k T_{o}\right)
$$

which is of the dual form of the control law that the "multirateinput controller" employs [8], [9].

Suppose we set the output multiplicities $\left(N_{1}, \cdots, N_{p}\right)$ to the minimum values satisfying ( 30$)$

$$
N_{i}=m_{i} \quad(i=1, \cdots, p)
$$

to obtain a stable controller. Then, the dynamical order of the controller remains the same $(=m)$ as the case of (26), but the sum $\bar{N}$ of the output multiplicities increases by $m$. The following lemma exhibits a sort of "consistency" between two design methods based on Theorems 1 and 2 (cf. [23] for the proof of the lemma).

Lemma 3: Suppose that the augmented system (19) is observable. Then for any OIV $\left(n_{1}, \cdots, n_{p}\right)$ of the pair $(A, C)$, there exists an OIV $\left(m_{1}, \cdots, m_{p}\right)$ of the augmented system such that $m_{i}$ $\geqq n_{i}(i=1, \cdots, p)$. Conversely, for any $\operatorname{OIV}\left(m_{1}, \cdots, m_{p}\right)$ of the augmented system, there exists an OIV $\left(n_{1}, \cdots, n_{p}\right)$ of the pair $(A, C)$ such that $n_{i} \leqq m_{i}(i=1, \cdots, p)$.

\section{Poles of the Closed-Loop System and Strong STABILIZABILITY}

Let us consider the poles of the closed-loop system shown in Fig. 2. From (7), we obtain

$$
\boldsymbol{x}\left(\overline{k+1} T_{o}\right)=\hat{A} \boldsymbol{x}\left(k T_{o}\right)+\hat{B} u\left(k T_{o}\right)
$$

where

$$
\begin{gathered}
\hat{A}=\exp \left(A T_{o}\right) \\
\hat{B}=\int_{0}^{T_{o}} \exp (A t) B d t .
\end{gathered}
$$

It follows from (10), (20), (24) [or (31)], (25), and (36) that the closed-loop system is described by

$$
\left(\begin{array}{l}
\boldsymbol{x}\left(\overline{k+1} T_{o}\right) \\
\boldsymbol{u}\left(\hat{k+1} T_{o}\right)
\end{array}\right)=\left(\begin{array}{cc}
\hat{A} & \hat{B} \\
-F \hat{A} & -F \hat{B}
\end{array}\right)\left(\begin{array}{l}
x\left(k T_{o}\right) \\
\boldsymbol{u}\left(k T_{o}\right)
\end{array}\right) .
$$

Applying the similarity transformation by the matrix

$$
\left(\begin{array}{cc}
I & 0 \\
-F & I
\end{array}\right)
$$

[25], we obtain

$$
\left(\begin{array}{l}
x\left(\overline{k+1} T_{o}\right) \\
z\left(\overline{k+1} T_{o}\right)
\end{array}\right)=\left(\begin{array}{cc}
\hat{A}-\hat{B} F & \hat{B} \\
0 & 0
\end{array}\right)\left(\begin{array}{l}
x\left(k T_{o}\right) \\
z\left(k T_{o}\right)
\end{array}\right)
$$

where

$$
\boldsymbol{z}\left(k T_{o}\right)=\boldsymbol{x}\left(k T_{o}\right)+F \boldsymbol{u}\left(k T_{o}\right) .
$$

Therefore, the closed-loop poles can be divided into two groups: $n$ poles which are the eigenvalues of the matrix $\hat{A}-\hat{B} F$ and $m$ poles located at the origin. This assures us of the important but nontrivial fact that the closed-loop system is stable if and only if the equivalently realized state feedback $F$ makes the "ideal" regulator $\hat{A}-\hat{B} F$ stable.

From the above consideration and from Theorem 2, we can derive an important theorem on the strong stabilizability [2], [3] of an unstable plant, i.e., the stabilizability using an asymptotically stable controller.

Theorem 3: A controllable and observable plant $(A, B, C)$ which satisfies the condition (29) is strongly stabilizable by a multirate-output controller (20) with almost any frame period $T_{0}$.

Proof: Since the pair $(\hat{A}, \hat{B})$ given by (37) and $(38)$ is controllable for almost every frame period $T_{0}[26]$, there exists a state feedback gain $F$ such that $\hat{A}-\hat{B} F$ is stable. If we note that the closed-loop system with the controller (20) is stable if and only if the equivalently realized state feedback $F$ of (31) makes $\hat{A}-$ $\hat{B} F$ stable, the result of the theorem follows by choosing a stable matrix $M$ of (32).

Q.E.D.

Note that the required condition (29) is equivalent to the following three conditions:

a) $m \leqq p$, i.e., the plant has at least as many outputs as inputs,

b) the plant is nondegenerate [27],

c) the plant has no invariant zeros [28] at the origin

In particular, if the plant is a controllable and observable singleinput single-output system, the conditions a)-c) reduce to the sole condition

$\left.c^{\prime}\right)$ the plant has no (blocking) zeros at the origin,

because the conditions a) and b) are always satisfied [27] and because the blocking zeros [29] and the invariant zeros are the same in this case.

On the other hand, it is well known that an unstable plant is strongly stabilizable by the conventional finite-dimensional linear time-invariant controllers if and only if it possesses the parity interlacing property (abbreviated as p.i.p.) [2], [3]. The p.i.p. is the condition on the location of real unstable poles and real unstable blocking zeros of the plant, and is clearly different from the conditions a)-c). In particular, as far as a single-input singleoutput plant is concerned, the condition $c^{\prime}$ ) is a very mild restriction as compared to the p.i.p. in the point that the location of the unstable poles of the plant is irrelevant. Actually, there is a large class of unstable plants which do not possess the p.i.p. and are still strongly stabilizable by the proposed multirate sampleddata controllers.

\section{EXAMPLES}

Two examples are studied in this section. The first example is a stable scalar plant. Concerning this plant, the standard procedure of designing MROC's and the possibility of obtaining a type-1 


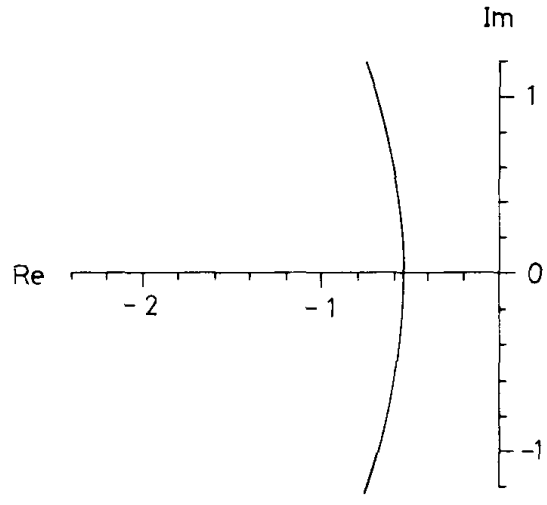

Fig. 3. Nyquist diagram of (Design 1-1).

servo system by appropriately choosing the controller's state transition matrix will be shown. The second example is an unstable one-input two-output plant which does not satisfy the p.i.p. condition. Concerning this plant, it will be exampled that we can construct a control system with satisfactory stability margins by using a stable MROC if the output multiplicities are increased appropriately.

Example 1: Consider the controllable and observable scalar plant with

$$
A=\left(\begin{array}{rrr}
0 & 1 & 0 \\
0 & 0 & 1 \\
-6 & -8 & -5
\end{array}\right), B=\left(\begin{array}{l}
0 \\
0 \\
1
\end{array}\right), C=\left[\begin{array}{lll}
10 & 7 & 1
\end{array}\right] .
$$

The transfer function is

$$
\frac{(s+2)(s+5)}{\left\{(s+1)^{2}+1\right\}(s+3)}
$$

and this plant satisfies (29)

To start the design we have to choose the design parameters. Here, let us choose the following:

$$
\text { frame period } T_{o}=0.2 \text {, }
$$

closed-loop poles $0.56 \pm 0.2 j$ and 0.65 .

The state feedback $F$ which assigns the above closed-loop poles should be calculated by using the pole-assignment routine. The result is

$$
F=\left[\begin{array}{lll}
10.600 & 9.8352 & 1.9354
\end{array}\right] .
$$

In the following, let us try two design methods: the method to choose the output multiplicities equal to the OIV of the plant, and the method to choose the output multiplicities equal to the OIV of the augmented system.

(Design 1-I): Since the plant has the observability index 3 , let the output multiplicity be $N_{1}=3$. Then, the matrix $H$ is uniquely determined by $(27)$ as

$$
H=\left[\begin{array}{lll}
24.817 & -59.188 & 35.880
\end{array}\right] .
$$

Therefore, by (25), we obtain

$$
M=H \hat{G}=0.74587
$$

which implies that the obtained controller itself is also stable. The Nyquist diagram considered at the plant input (or equivalently, at the plant output) is as shown in Fig. 3, which indicates that the gain margin and the phase margin are about $+5.4 \mathrm{~dB}$ and $51^{\circ}$, respectively.

(Design 1-2): Since the augmented system has the observability
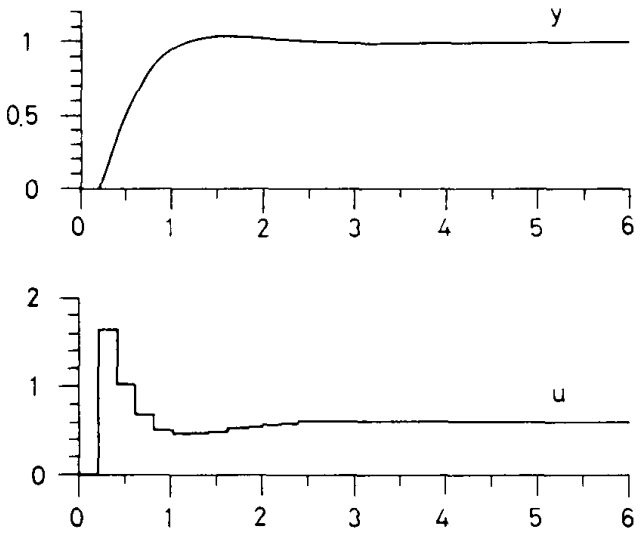

Fig. 4. Indicial response of (Design 1-2).

index 4 , let the output multiplicity be $N_{1}=4$. In order to construct a type-1 robust servo-control system, let us set $M=1$ in (32). Then, the matrix $H$ is uniquely determined by solving (33) as

$$
H=\left[\begin{array}{llll}
-147.73 & 527.35 & -627.57 & 249.60
\end{array}\right] .
$$

The resulting closed-loop system has the gain margin about +4.5 $\mathrm{dB}$ and the phase margin about $40^{\circ}$. The indicial response of the closed-loop system under the initial conditions $x(0)=[0,0,0]^{T}$ and $u(0)=0$ is as shown in Fig. 4, where $-\hat{y}$ is replaced by $\hat{e}(e$ denotes the error $r-y ; r$ : reference) in the control law (20).

Example 2: Consider the fourth-order plant with

$$
\begin{gathered}
A=\left(\begin{array}{rrrr}
2 & 0 & 0 & 0 \\
2 & -1 & 0 & 0 \\
-1 & 0 & -3 & 0 \\
1 & 0 & 0 & -2
\end{array}\right), B=\left(\begin{array}{r}
1 \\
2 \\
-1 \\
1
\end{array}\right), \\
C=\left(\begin{array}{llll}
0 & 1 & 1 & 0 \\
0 & 0 & 0 & 1
\end{array}\right) .
\end{gathered}
$$

This plant is controllable and observable, and satisfies (29). The transfer function matrix is

$$
\left(\begin{array}{c}
\frac{(s-1)(s+5)}{(s-2)(s+1)(s+3)} \\
\frac{(s-1)}{(s-2)(s+2)}
\end{array}\right) \text {. }
$$

Equation (50) implies that this plant has a blocking zero at $s=1$ and an unstable pole at $s=2$. Namely, the plant does not possess the p.i.p. in the continuous-time sense [2], [3].

Let the frame period $T_{o}=0.2$, and let us determine the desirable state feedback $F$ by solving the optimal regulation problem. For the performance index

$$
J=\sum_{k=0}^{\infty}\left\{y^{T}\left(k T_{o}\right) Q y\left(k T_{o}\right)+u\left(k T_{o}\right)^{2}\right\}
$$

$$
Q=\operatorname{diag}[5,5]
$$

the optimal state feedback $F$ is given as

$$
F=\left[\begin{array}{llll}
4.3873 & 1.5444 \times 10^{-3} & 1.7478 \times 10^{-1} & 8.5964 \times 10^{-2}
\end{array}\right] .
$$

The above state feedback is equivalently realized by the multirateoutput controller as follows. Also for this example, let us try the two design methods.

(Design 2-1): Since the plant has OIV $(2,2)$, let the output multiplicities be $N_{1}=N_{2}=2$. Then, the matrix $H$ is uniquely 


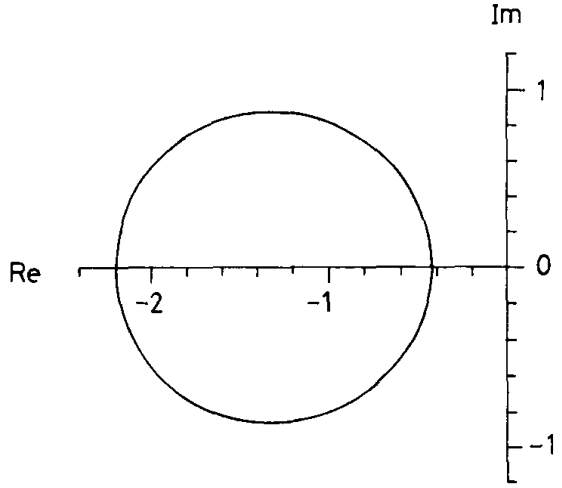

Fig. 5. Nyquist diagram of (Design 2-2).
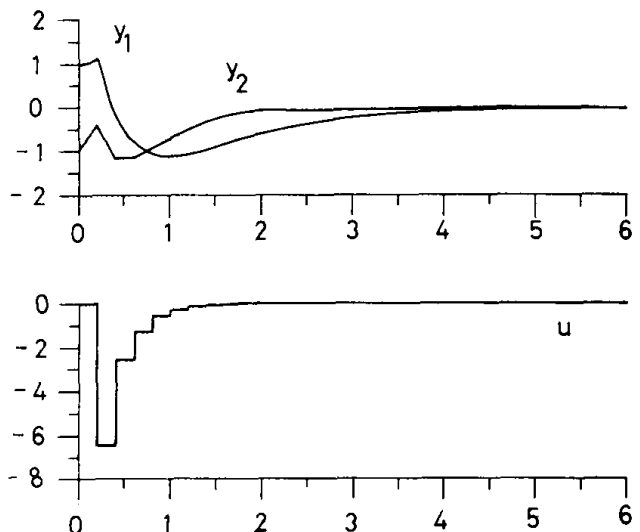

Fig. 6. Transient response of (Design 2-2).

determined by (27) as

$$
H=\left[\begin{array}{lll}
5.2346 \times 10^{-1} & -5.7712 \times 10^{-1}-53.59465 .530
\end{array}\right] .
$$

Therefore, we obtain

$$
M=H \hat{G}=5.1386>1
$$

which implies that the obtained controller is unstable. Analysis using a Nyquist diagram shows that the gain margins considered at the plant input are about $+0.86 \mathrm{~dB}$ and $-1.2 \mathrm{~dB}$, and the phase margin is about $6^{\circ}$.

(Design 2-2): Since the augmented system has OIV $(3,2)$, let the output multiplicities be $N_{1}=3$ and $N_{2}=2$, and let us design a stable controller. By setting $M=0$ in (32), the multirate sampled-data nondynamic output feedback controller (34) is obtained, where the matrix $H$ is uniquely determined by solving (33) as

$$
H=\left[\begin{array}{lllll}
899.43 & -2059.1 & 1173.3 & 245.21 & -299.44
\end{array}\right] .
$$

The Nyquist diagram considered at the plant input is as shown in Fig. 5. This indicates that the gain margins are about $+7.4 \mathrm{~dB}$ and $-6.9 \mathrm{~dB}$, and the phase margin is about $41^{\circ}$. These stability margins are exactly the same as those of the "ideal" regulator with the state feedback (53). The transient response of the resulting closed-loop system is as shown in Fig. 6, where the initial conditions are $\boldsymbol{x}(0)=[1,1,0,-1]^{T}$ and $u(0)=0$.

\section{CONCLUSIONS}

A new class of controllers using the multirate sampling of the plant output is proposed. From the theoretical point of view, the most attractive advantage of the proposed controllers exists in that, under the assumptions of Theorem 2 , the state transition matrix of the controller itself can be arranged to have any prescribed value without changing the state feedback to be realized. Use can be made of this advantage to expand the stability margins and to improve the steady-state characteristics of the closed-loop system. Furthermore, a wide class of unstable plants without the parity interlacing property can be strongly stabilized by the proposed controllers. On account of these theoretical bases, the proposed controllers are expected to be successfully applied to the industrial problems.

In the proposed controllers, the values of the control inputs are determined based on the output data obtained during the last one frame period. It is possible to generalize the control law, i.e., to determine the inputs based on the output data obtained during the last $L(\geqq 2)$ frame periods. By using such generalized control laws, we can realize the state feedback controller with smaller output multiplicities, and, so, relax the requirement for quick observations. In particular, if we choose $L=n$ where $n$ is the order of the plant, the suggested control law would be equivalent to the Mita et al. controller of [12]. Study on advantages and disadvantages of such generalized control laws is a future problem.

\section{APPENDIX}

(Proof of Lemma 1)

Multiply (11) by exp $\left(A T_{o}\right)$ from the right to obtain

$$
\hat{C} \exp \left(A T_{o}\right)=\left(\begin{array}{c}
c_{1} \\
: \\
c_{1} \exp \left\{A\left(N_{1}-1\right) T_{1}\right\} \\
: \\
c_{p} \\
: \\
c_{p} \exp \left\{A\left(N_{p}-1\right) T_{p}\right\}
\end{array}\right\} .
$$

Observe that there exists a series of elementary row transformations denoted by the matrix $S$ such that

$$
S \hat{C} \exp \left(A T_{o}\right)=\left(\begin{array}{c}
c_{1} \\
: \\
c_{1}\left\{\exp \left(A T_{1}\right)-I\right\}^{N_{1}-1} \\
: \\
c_{p} \\
: \\
c_{p}\left\{\exp \left(A T_{p}\right)-I\right\}^{N_{p}-1}
\end{array}\right) .
$$

Since matrices $S$ and exp $\left(A T_{o}\right)$ are nonsingular, it is enough to show that $S \hat{C} \exp \left(A T_{o}\right)$ has full column rank. Deleting appropriate rows of the matrix $S \hat{C} \exp \left(A T_{0}\right)$, we obtain the square matrix

$$
\left(\begin{array}{c}
c_{1} \\
: \\
c_{1}\left\{\exp \left(A T_{1}\right)-I\right\}^{n_{1}-1} \\
: \\
c_{p} \\
: \\
c_{p}\left\{\exp \left(A T_{p}\right)-I\right\}^{n_{p}-1}
\end{array}\right) .
$$


Apparently, the rank of the matrix (A3) is the same as that of the matrix

$$
\left(\begin{array}{c}
c_{1} \\
: \\
c_{1}\left\{\exp \left(A T_{1}\right)-I\right\}^{n_{1}-1} / T_{1}^{n_{1}-1} \\
: \\
c_{p} \\
: \\
c_{p}\left\{\exp \left(A T_{p}\right)-I\right\}^{n_{p}-1} / T_{p}^{n_{p}-1}
\end{array}\right],
$$

and this matrix goes to

$$
\left(\begin{array}{c}
c_{1} \\
: \\
c_{1} A^{n_{1}-1} \\
: \\
c_{p} \\
: \\
c_{p} A^{n_{p}-1}
\end{array}\right)
$$

as $T_{o}$ goes to 0 . Since the determinant of a matrix is a continuous function of its entries, the determinant of (A4) goes to that of (A5), which is nonzero by the assumption. This implies that the determinant of (A3) is nonzero for sufficiently small $T_{o}$. Observing that the determinant of (A3) is an analytic function of $T_{o}$ since so is each entry of (A3), it follows that the determinant of (A3) is nonzero for almost every $T_{o}$. The required result follows readily.

Q.E.D.

\section{REFERENCES}

[1] W. M. Wonham, Linear Multivariable Control: A Geometric Approach. New York: Springer-Verlag, 1974.

[2] D. C. Youla, J. J. Bongiorno, Jr., and C. N. Lu, "Single-loop feedback-stabilization of linear multivariable dynamical plants," Automatica, vol. 10, pp. 159-173, 1974

[3] M. Vidyasagar, Control System Synthesis: A Factorization Approach. Cambridge, MA: M.I.T. Press, 1985

[4] A. B. Chammas and C. T. Leondes, "On the design of linear timeinvariant systems by periodic output feedback: Part I. Discrete-time pole assignment," Int. J. Contr., vol. 27, pp. 885-894, 1978.

[5] _ _ "On the design of linear time-invariant systems by periodic output feedback: Part II. Output feedback controllability," Int. J. Contr., vol. 27, pp. 895-903, 1978.

[6] ___. "Pole assignment by piecewise constant output feedback," Int J. Contr., vol. 29 , pp. $3 \mathrm{i}-38,1979$

[7] _-., "On the finite time control of linear systems by piecewise constant output feedback," Int. J. Contr., vol. 30, pp. 227-234, 1979 .

18] M. Araki and T. Hagiwara, "Pole assignment by multirate sampleddata output feedback," in Proc. 24th IEEE Conf. Decision Contr. 1985 , pp. 189-193

[9] M. Araki and T. Hagiwara, "Pole assignment by multirate sampleddata output feedback," Int. J. Contr., vol. 44, pp. 1661-1673, 1986.

[10] J. P. Greschak and G. C. Verghese, "Periodically varying compensation of time-invariant systems," Syst. Contr. Lett., vol. 2, pp. 88-93, 1982.

[11] P. P. Khargonekar, K. Poolla, and A. Tannenbaum, "Robust control of linear time-invariant plants using periodic compensation," IEEE Trans. Automat. Contr., vol. AC-30, pp. 1088-1096, 1985

[12] T. Mita, B. C. Pang, and K. Z. Liu, "Design of optimal strongly stable digital control systems and application to output feedback control of mechanical systems," Int. J. Contr., vol 45, pp. 2071-2082, 1987.

[13] G. M. Kranc, "Input-output analysis of multirate feedback system," IRE Trans. Automat. Contr., vol. AC-3, pp. 21-28, 1957.
[14] R. E. Kalman and J. E. Bertram, "A unified approach to the theory of sampling systems," J. Franklin Inst., vol. 267, pp. 405-436, 1959 T. C. Coffey and I. J. Williams, "Stability analysis of multiloop. multirate sampled systems," AIAA J., vol. 4, pp. 2178-2190, 1966.

[16] E. I. Jury, "A note on multirate sampled-data systems," IEEE Trans. Automat. Contr., vol. AC-12, pp. 319-320, 1967.

[17] W. H. Boykin and B. D. Frazier, "Multirate sampled-data systems analysis via vector operators," IEEE Trans. Automat. Contr., vol. AC-20, pp. 548-551, 1975.

[18] R. A. Meyer and C. S. Burrus, "Design and implementation of multirate digital filters," IEEE Trans. Acoust., Speech, Signal Processing, vol. ASSP-24, pp. 53-58, 1976.

[19] C. H. Lu and S. C. Gupta, "Multirate digital filters," Int. J. Syst. Sci., vol. 10, pp. 605-620, 1979.

[20] M. Araki, K. Yamamoto, and B. Kondo, "Multivariable multirate sampled-data systems: Symmetric coordinate expression, stability, and generalized Gershgorin bands," presented at the U.S.-Japan Seminar on Recent Advances in Algebraic System Theory, Gainesville, FL, Apr. 1983.

[21] M. Araki and K. Yamamoto, "Multivariable multirate sampled-data systems: State-space description, transfer characteristics, and Nyquist criterion," IEEE Trans. Automat. Contr., vol. AC-31, pp. 145-154, 1986.

[22] R. E. Kalman, "Kronecker invariants and feedback," in Proc. Conf. Ordinary Differential Equations, Math. Research Center, Naval Research Labs., Washington, DC, 1971, pp. 459-471.

[23] D. G. Luenberger, "Canonical forms for linear multivariable systems," IEEE Trans. Automat. Contr, vol. AC-12, pp. 290-293, 1967

[24] H. W. Smith and E. J. Davison, “Design of industrial regulators Integral feedback and feedforward control," Proc. IEE, vol. 119, no. 8 , pp. $1210-1216,1972$

[25] T. Mita, "Optimal digital feedback control systems counting computation time of control laws," IEEE Trans. Automat. Contr., vol. AC 30 , pp. $542-548,1985$

[26] R. E. Kalman, Y. C. Ho, and K. S. Narendra, "Controllability of linear dynamical systems," Contributions to Differential Equations, vol. 1, no. 2, pp. 189-213, 1962 .

[27] E. J. Davison and S. H. Wang, "Properties and calculation of transmission zeros of linear multivariable systems, "Automatica, vol. 10. pp. 643-658, 1974 .

[28] A. G. J. MacFarlane and N. Karcanias, "Poles and zeros of linear multivariable systems: A survey of the algebraic, geometric and complex-variable theory," Int. J. Contr., vol. 24, pp. 33-74, 1976

[29] P. G. Ferreira and S. P. Bhattacharyya, "On blocking zeros," IEEE Trans. Automat. Contr., vol. AC-22, pp. 258-259, 1977.

[30] M. Araki and T. Hagiwara, "Several approaches to the design of multirate-output controllers," to be published.

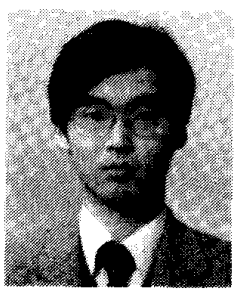

Tomomichi Hagiwara was born in Osaka, Japan, on March 28, 1962. He received the B.E. and M.E. degrees, both in electrical engineering, from Kyoto University, Kyoto, Japan, in 1984 and 1986, respectively.

Since 1986 he has been an Instructor in the Department of Electrical Engineering, Kyoto University. His research interests are in digital control systems, multivariable control systems, and system theory.

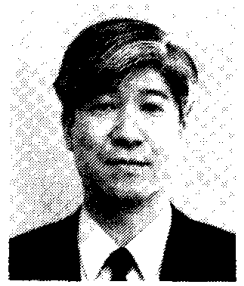

Mituhiko Araki (M'88) was born in Tokyo, Japan, on September 25, 1943. He received the B.E. M.E., and Ph.D. degrees, all in electronic engineering, from Kyoto University, Kyoto, Japan, in 1966, 1968, and 1971, respectively.

Since 1971 he has been with the Department of Electrical Engineering, Kyoto University, where he is currently a Professor. His research interests are in digital control, stability theory, large-scale systems. nonlinear systems, and applications. 